\title{
Impacto del riesgo climático en las actividades económicas. Análisis del sector líneas aéreas
}

\section{lgnacio López Domínguez, Rebeca Velázquez Boeta}

Facultad de Ciencias Económicas y Empresariales Universidad Complutense de Madrid, España

Universidad Nacional Autónoma de México (UNAM), México

El propósito de esta investigación es analizar el impacto negativo que tiene el comportamiento del clima en los sectores económicos con el fin de minimizar dicho impacto a través de la implementación de mecanismos adaptativos para generar certidumbre en los rendimientos de las empresas. Para ello, se describen las vulnerabilidades de cada sector económico (primario, secundario y terciario); posteriormente, el artículo se centra en el sector servicios, concretamente en el sector de líneas áreas, para lo cual se estudia la correlación entre la adaptación y los procesos de mitigación con el fin de disminuir el impacto negativo del riesgo climático. Las principales conclusiones son que todas las actividades tienen una gran sensibilidad ante el clima debido a la interrelación que presentan las actividades productivas entre sí. Por ello, es necesaria la adaptación ante dichos cambios climáticos con el fin de poder minimizar la vulnerabilidad (sensibilidad) de las actividades, ya que, en el largo plazo, el impacto será mayor. La principal aportación de esta investigación consiste en facilitar y mejorar la capacidad de enfrentar los riesgos climáticos, pues, al identificar de manera más certera la vulnerabilidad ante el cambio climático, los rendimientos tendrán mejor certidumbre y, por lo tanto, las empresas tendrán más posibilidades de supervivencia.

Palabras clave: vulnerabilidad, riesgo climático, administración del riesgo, capacidad adaptativa

\section{Impact of climate risk on economic activities. Analysis of the airlines sector}

The purpose of this research is to analyze the negative impact of climate behavior on economic sectors in order to minimize this impact through the implementation of adaptive mechanisms to generate certainty in the performance of companies. With this goal, first we present a brief description of the vulnerabilities of each economic sector (primary, secondary and tertiary) in general terms and, subsequently, focus on the services sector, the sector lines, studying the correlation between adaptation and the processes of mitigation in order to reduce the negative impact of climate risk. Our main conclusion is that all the activities have a great sensitivity to the climate due to the interrelation that the productive activities present to each other. Adaptation to these climatic changes is therefore necessary in order to minimize the vulnerability (sensitivity) of the activities since in the long term the impact will be greater. The value of this paper is to facilitate or improve the capacity to face weather risks because the better the vulnerability to climate change is identified, the better certainty will be the companies' returns, and their survival.

Keywords: vulnerability, weather risk, risk management, adaptive capacity 


\section{Impacto do risco climático nas atividades econômicas. Análise do setor aéreo}

O objetivo desta pesquisa é analisar o impacto negativo que o comportamento climático tem sobre os setores econômicos, a fim de minimizar esse impacto por meio da implementação de mecanismos adaptativos para gerar certeza no desempenho das empresas. Para isso, são descritas as vulnerabilidades de cada setor econômico (primário, secundário e terciário) para, posteriormente, focalizar o setor de serviços, áreas de linhas setoriais, estudando a correlação entre os processos de adaptação e mitigação, a fim de reduzir $\mathrm{O}$ impacto negativo do risco climático. As principais conclusões são que todas as atividades têm uma grande sensibilidade ao clima devido à inter-relação que as atividades produtivas apresentam entre si. Assim, é necessária a adaptação a essas mudanças climáticas, a fim de minimizar a vulnerabilidade (sensibilidade) das atividades, pois, a longo prazo, o impacto será maior. A principal contribuiçâo é facilitar ou melhorar a capacidade de enfrentar os riscos climáticos, porque quanto melhor for identificada a vulnerabilidade às mudanças climáticas, maior será a certeza dos rendimentos e, portanto, da sobrevivência das empresas.

Palavras-chave: vulnerabilidade, risco climático, gerenciamento de risco, capacidade adaptativa

\section{Introducción}

Las actividades humanas han ido cambiando a lo largo del tiempo debido al desarrollo económico e industrial, que han traído como consecuencia la explotación de los bosques, las tierras y demás recursos naturales. Estos excesos han transformado la conducta del clima en el planeta. Dicho efecto se conoce como cambio climático, pues supone el aumento de la temperatura del planeta y el nivel del mar, y agrava fenómenos naturales como huracanes, lluvias, heladas, nevadas, etcétera (Villalba Fonfría, 2008). El cambio climático contribuye a complicar el panorama de la degradación ecológica y ambiental a escala global y trae consigo un incremento de diferentes riesgos para las poblaciones humanas en distintos ámbitos (Ortiz-Paniagua \& Pérez, 2017). La agricultura es vulnerable a los fuertes cambios socioeconómicos que se dan a partir del proceso de globalización económica y es también altamente sensible a las variaciones climáticas (Conde, Ferrer, Gay \& Araujo, 2004). Adicionalmente, estudios relacionados a la agricultura consideran que esta actividad es extremadamente vulnerable en los países en desarrollo (O’Brien \& Leichenko, 2000).
Las consecuencias del cambio climático impactan negativamente en las actividades de la sociedad y de la economía al perturbar la calidad de vida (Duarte Cueva, 2014).

Para muchas empresas es un requisito indispensable tener una estrategia para controlar los riesgos a los que se enfrentan, pues, de esta forma, aseguran su estabilidad operativa y financiera. Los riesgos en que más se han enfocado las variaciones en el clima también pueden provocar impactos negativos en las previsiones de costos y gastos de las empresas (Monjas, 2010). Por ello, se vuelve necesario desarrollar estrategias ambientales innovadoras que permitan transferir el riesgo climático, ya sea a través de seguros contra catástrofes o a través de diferentes estrategias financieras, pues se ha generado un creciente interés en los mercados de seguros meteorológicos. Esto forma oportunidades de innovación en modelos de negocio en distintos sectores, como los de salud y seguridad. Tales oportunidades no han sido explotadas en su totalidad y, de hacerlo, se cambiaría el modo de combatir el cambio climático y se incrementaría la concientización para frenar esta problemática. 


\section{Revisión de la literatura e hipótesis}

Para muchas empresas es un requisito indispensable tener una estrategia para controlar los riesgos a los que se enfrentan, pues implica que aseguren su estabilidad operativa y financiera. Los riesgos en que se han enfocado más son los que impactan directa y rápidamente a sus resultados, como el riesgo de mercado (tipo de cambio, tasas de interés, etc.), el crediticio (préstamos o financiamientos) y de liquidez (compra y venta de activos). Sin embargo, cada vez cobra mayor importancia el riesgo generado por variaciones en el clima, que provocan fuertes impactos negativos en las previsiones de costos y gastos de las empresas (Monjas, 2010).

Los riesgos climáticos que afectan las actividades económicas son considerados como catastróficos y no catastróficos, ya que dependen del grado de daño que generan. El riesgo climático catastrófico consiste en eventos con poca probabilidad de ocurrencia que causen daños financieros masivos; entre ellos, se encuentran las inundaciones, los huracanes y los tornados, entre otros fenómenos naturales. El clima no catastrófico se relaciona con desviaciones menores del clima habitual. Actualmente, las empresas solo han tomado en cuenta el riesgo catastrófico a través de la contratación de seguros contra desastres naturales. No obstante, no han tomado en cuenta que el riesgo no catastrófico afecta el desempeño de estas; es decir, se tornan sensibles al comportamiento del clima, ya que las desviaciones estacionales en el comportamiento del clima generan incertidumbre en los flujos de efectivo futuros.

La sensibilidad o la exposición al clima se define como la propensión de las ventas, y los procesos productivos o los costos a elementos meteorológicos como la temperatura, la luz solar, las precipitaciones, las nevadas, el viento, etc. (Štulec, 2017). Esto implica que, si la producción de un sector determinado presenta variaciones que son causadas por los cambios en el comportamiento del clima, el sector es sensible al clima. Diversas investigaciones sostienen que las empresas de todos los sectores económicos (primarios, secundarios y terciarios) presentan sensibilidad o exposición al comportamiento del clima. Su importancia varía de acuerdo al sector y al área geográfica a la que pertenezcan (Wang, Ball, Nehring, Williams \& Chau, 2017), es decir, el impacto del clima en cada sector y empresa varía de acuerdo al área donde realicen sus actividades productivas; incluso, la sensibilidad puede variar significativamente entre dos empresas en el mismo país. El impacto climático se agrava conforme el comportamiento del clima se intensifica, y afecta los costos y gastos previstos por las empresas, pues conlleva a la incertidumbre de los rendimientos. Además, la vulnerabilidad o sensibilidad que presentan las actividades económicas frente al clima depende tanto de la exposición a los cambios en el clima como de la capacidad del sistema afectado para adaptarse (Smit, Ford \& Wandel, 2006).

Por las razones expuestas, se vuelve necesario implementar medidas correctivas o adaptativas (estrategias de administración del riesgo) para facilitar la identificación de la correlación que existe entre las actividades económicas y el comportamiento del clima. Esto tiene el fin de mejorar la capacidad de enfrentar los riesgos climáticos, pues, mientras mejor se identifique la vulnerabilidad ante el cambio climático, mejor certidumbre se tendrá sobre los rendimientos de las empresas. El desarrollo de innovaciones se ha enfocado en la solución de problemas sociales y económicos, cuyos impactos son percibidos a corto plazo. Se han dejado de lado a los problemas ambientales por tener un impacto a largo plazo. 
Se han realizado investigaciones en las que se estudia la posible mitigación del riesgo climático en las actividades económicas a través del desarrollo de enfoques sustentables. Tales estudios también persiguen el objetivo de mejorar la calidad de vida de la humanidad por medio de la preservación de la calidad del ambiente, así como por medio de la implementación de herramientas enfocadas en la cobertura del riesgo climático. Las investigaciones realizadas por Hong, Weikai y Jiangmin (2017) y Hsiang (2016) sostienen la existente relación entre los riesgos climáticos y las actividades económicas; además de medir la sensibilidad frente al comportamiento del clima, se apoyan en modelos econométricos para sustentar el impacto negativo existente. Por su parte, Schlenker y Taylor (2019) han estudiado los modelos climáticos y su comportamiento en el mundo financiero a través de tendencias y proyecciones. Asimismo, Monjas (2010) realizó un estudio sobre el impacto que tiene el clima en los diferentes sectores económicos. Para ello, realizó una simulación de escenarios para el sector energético, lo que lo lleva a afirmar la importancia de la implementación de derivados climáticos como cobertura del riesgo climático y como estrategia innovadora para la administración del riesgo. Por último, Whalley y Yuan (2009) realizaron un análisis sobre las implicaciones del calentamiento global a mediano y largo plazo para la evolución de las estructuras financieras globales, implicaciones que generan innovaciones en el mercado financiero. Entre ellas, se pueden destacar las siguientes: la expansión del mercado de seguros climáticos, la facilitación de la acumulación de activos y la diversificación de riesgos asociados al cambio climático.

Al haber realizado un análisis detallado de las investigaciones anteriormente descritas, se puede observar que el panorama acerca del estudio sobre el impacto del clima en las actividades económicas se enfoca básicamente en el sector agrícola y el energético, seguidos del sector de alimentos. Asimismo, las investigaciones tienden a registrar los resultados positivos. Por esto, se torna al tamente factible el estudio del impacto del clima en los sectores económicos con el fin de implementar medidas de adaptación que minimizan las vulnerabilidades existentes en dichos sectores ante el cambio climático; de esta forma, se tendrá un panorama de certidumbre frente a los resultados de las empresas.

La hipótesis de esta investigación consiste en que la identificación de las vulnerabilidades de las actividades económicas frente al clima permite identificar la correlación (sensibilidad) que existe entre estas. Esto posibilita minimizar el impacto negativo a través de la implementación de medidas de adaptación como estrategias de mitigación del riesgo climático.

\subsection{Análisis de vulnerabilidad}

De acuerdo con el modelo de vulnerabilidad y adaptación de Smit et al. (2006) (ver figura 1), a través del análisis de exposición y sensibilidad que presenta un sistema o comunidad (en este caso, la empresa), se desarrollan métodos o estrategias para administrar el riesgo climático con el fin de facilitar la capacidad adaptativa en cuestión y minimizar el impacto negativo que existe entre estas. En otras palabras, la vulnerabilidad está en función a la sensibilidad de la exposición de las empresas a los efectos del cambio climático y su capacidad de adaptación para hacer frente a dicha exposición.

En el análisis de vulnerabilidades, se encuentran tres componentes principales:

- Exposición: se define como el tipo y grado (o naturaleza) al que un sistema está expuesto o que es afectado por variaciones climáticas significativas.

Contabilidad y Negocios (15) 29, 2020 / ISSN 1992-1896 
Figura 1. Modelo de vulnerabilidad

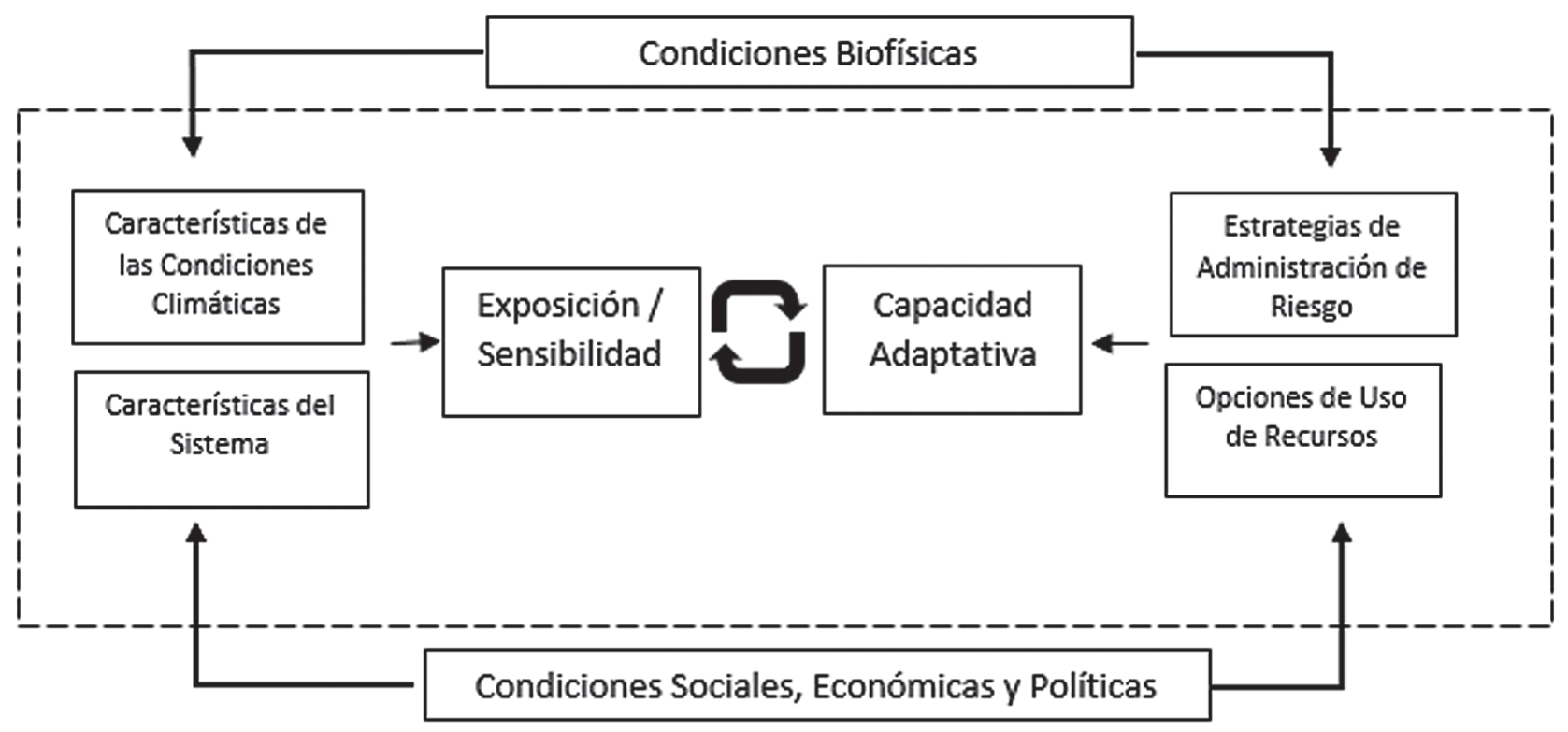

Procesos que operan a escalas más allá del sistema de interés.

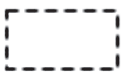

Sistemas de Interés, actividad, sector, comunidad o región.

Fuente: Smit et al. (2006).

Depende tanto de las características de las condiciones climáticas como de la naturaleza de la comunidad en cuestión. Las características de las condiciones relacionadas con el clima incluyen la magnitud, la frecuencia, la dispersión espacial, la duración, la velocidad de inicio, el tiempo y el espaciado temporal de las condiciones. Por esta razón, la exposición es claramente dinámica, pues cambia a medida que la comunidad cambia sus características en relación con las condiciones climáticas y también cambia a medida que cambian los estímulos (Smit et al., 2006).
- Sensibilidad: se explica como el grado en que un sistema es afectado por el cambio climático positiva o negativamente. La sensibilidad refleja la susceptibilidad de las personas y las comunidades a las condiciones que representan riesgos, incluidas las asociadas con el cambio climático (Smit et al., 2006).

- Capacidad adaptativa: se describe como la capacidad o el potencial de un sistema o comunidad para abordar, planificar o adaptarse a la sensibilidad de la exposición; es decir, ajustarse al cambio climático. 
Incluye la variabilidad climática y los fenómenos extremos con el fin de moderar los daños potenciales, de beneficiarse de las oportunidades o de afrontar las consecuencias. La capacidad adaptativa implica una alteración de algo (sistema de interés, actividad, sector, comunidad o región) frente a algo (el estrés o estímulo relacionado con el clima). La descripción de una adaptación requiere una especificación de quién o qué se adapta, el estímulo para el cual se realiza la adaptación, y el proceso y la forma que toma (Smit et al., 2006).

El análisis de la vulnerabilidad se encuentra en función de las actividades que se realizan o se dejan de realizar. Estas decisiones conllevan a reducir o aumentar los impactos del cambio climático. Además, proporcionan información para diseñar e implementar medidas de adaptación específicas para disminuir la vulnerabilidad ante el cambio climático.

Las personas y comunidades han aprendido a modificar su comportamiento y su entorno para gestionar y aprovechar sus condiciones climáticas locales. En ese sentido, esta investigación está dirigida precisamente para la adaptación al cambio climático a través de la implementación de estrategias enfocadas en la cobertura del riesgo climático. Esta capacidad de adaptación refleja las opciones de uso de recursos y las estrategias de gestión de riesgos para prepararse, evitar o moderar, y recuperarse de los efectos de la exposición (Smit et al., 2006).

\subsection{Capacidad adaptativa}

La capacidad adaptativa se describe en dos vertientes: la primera trata sobre la evaluación de los impactos y las vulnerabilidades; y la otra, sobre el desarrollo y evaluación de las opciones de respuesta (mitigación o administración del riesgo climático). De esta forma, el análisis de vulnerabilidad se encuentra aunado al pro- ceso de adaptación al cambio climático (ver figura 2), ya que conlleva a evaluaciones de sensibilidades (vulnerabilidades) y al diseño de medidas de adaptación. Estas últimas están encaminadas a la minimización del impacto negativo del clima en los sectores económicos.

Figura 2. Proceso de adaptación

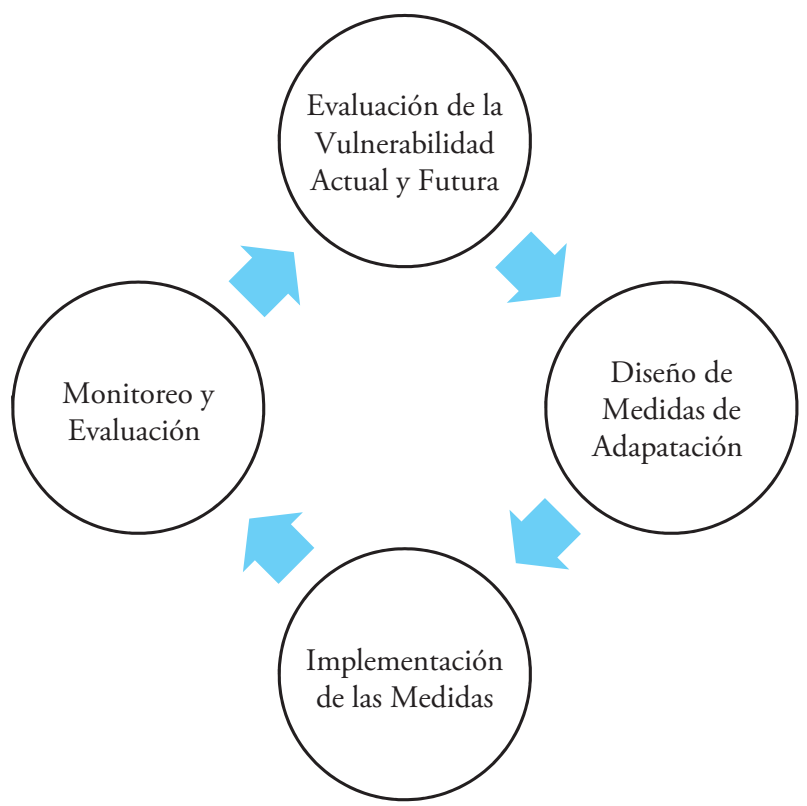

Fuente: Elaboración propia a partir de datos del Instituto Nacional de Ecología y Cambio Climático (2020).

El Programa de trabajo de Nairobi (United Nations Framework Convention on Climate Change [UNFCCC], 2007) presenta diferentes propuestas encaminadas a la orientación sobre áreas de trabajo respecto de la adaptación al cambio climático, que se pueden tomar como posibles herramientas para lograr este objetivo:

- Métodos e instrumentos: promueven el desarrollo de métodos y herramientas para la evaluación y diagnóstico de los impactos del cambio climático, la vulnerabilidad y las medidas y acciones de adaptación elegidas. 
- Datos y observaciones: suponen el apoyo con el manejo de datos y observaciones meteorológicas que permiten evaluar los impactos, la vulnerabilidad y adaptación al cambio climático.

- Modelización del clima y escenarios: promueve el desarrollo de escenarios y modelos del clima respecto del cambio climático con el fin de ayudar en la evaluación de los impactos, la vulnerabilidad y la selección de estrategias de adaptación.

- Riesgos climáticos y fenómenos extremos: implica el uso de información climática para evaluación, planeación e implementación de acciones que permitan la adaptación del cambio climático.

- Información socioeconómica: se utiliza para medir el impacto en el cambio climático y para establecer acciones de adaptación frente al cambio climático.

- Planificación y prácticas de la adaptación: implican la reducción de la vulnerabilidad y la adaptación a los impactos del cambio climático de la mejor forma.

- Investigación: fomenta el desarrollo y difusión de tecnología, conocimiento, y lecciones aprendidas en materia de adaptación.

- Tecnologías para la adaptación: supone la transferencia de tecnologías y conocimiento que promueven medidas para adaptarse al cambio climático eficientemente. La falta de tecnología impide que una nación sea capaz de implementar opciones de adaptación, pues se limita el rango de posibles respuestas.

El camino más seguro para la adaptación consiste en la construcción de escenarios futuros. Esto supone el establecimiento de posibles escenarios críticos con el fin de conocer momentos cruciales de acuerdo al riesgo al que se está expuesto, y al sector al que pertenece la empresa. Así, se podrá prever el comportamiento que se deberá tener en caso de que ocurra cualquier escenario. El siguiente paso es la elaboración de planes y estrategias de adaptación, los cuales deben basarse en los escenarios analizados con el fin de hacer eficiente el análisis. Estos planes deben contar con planes de prevención, de seguimiento y de contingencia. Por último, se deben gestionar los sistemas de información con el fin de generar transparencia y concientización de los procesos y planes implementados.

\section{Metodología}

En esta sección, se identificará y se describirá el impacto de las actividades que se realizan en cada sector económico ante el cambio climático. Se iniciará con la descripción de las vulnerabilidades de cada sector y, posteriormente, se identificará la correlación (sensibilidad) que existe entre las actividades económicas y el comportamiento del clima. Se analizará el impacto en el sector primario, secundario y terciario con el fin de encontrar las vulnerabilidades existentes en cada sector económico. De esta forma, se planteará una propuesta de gestión y enfrentamiento de dicho riesgo climático, con el fin de poder adaptarse ante los cambios en el clima.

\subsection{Descripciones de las vulnerabilidades de cada sector}

El cambio climático ha generado muchas controversias debido a las consecuencias que presenta en el planeta. El propósito de describir el impacto que tiene el clima sobre las actividades económicas consiste en identificar las vulnerabilidades de cada sector para generar medidas encaminadas para adaptarse al comportamiento del clima. 
- Sector primario: la importancia de este sector radica en que en este se producen alimentos, es decir, recursos naturales que son vitales para la sociedad, la industria y el ecosistema.

- Sector secundario: la importancia de este sector reside en el suministro de recursos; la cantidad y calidad de la producción de la industria para la sociedad; y el precio de los productores, es decir, el precio de abastecimiento y distribución de la producción.

- Sector terciario: la importancia de este sector consiste en la calidad de servicios suministrados a la sociedad y las actividades que se realizan en este.

\subsection{Exposición y sensibilidad del impacto del riesgo climático en sectores económicos}

En esta sección, se realiza un análisis sobre la sensibilidad que tienen las actividades correspondientes a cada sector económico con respecto al comportamiento del clima. Para ello, se mide la correlación que existe entre ambos factores con el fin de determinar el grado de vulnerabilidad que existe. El análisis de sensibilidad del impacto y exposición inicia con la estimación basada en datos históricos sobre cambios en la producción efectuada en cada sector económico; es decir, se analiza el producto interno bruto (PIB) de los últimos veinte años con el fin de dimensionar los ingresos que podrán recibir o no recibir las empresas pertenecientes a los sectores económicos por cada punto de desviación frente al comportamiento del clima. Para medir el clima, se utilizó el promedio de precipitación a nivel mundial por los últimos veinte años.

Se determinó una regresión lineal múltiple con el fin de estimar el comportamiento de la producción efectuada por cada sector económico con respecto a cambios en el comportamiento del promedio de precipitación mensual determinado por el Banco Mundial. La regresión múltiple toma en cuenta como variable dependiente el PIB de cada sector en particular expresado en variaciones porcentuales anuales. Las variables independientes están relacionadas con cada sector en particular y son descritas en la tabla 1 .

Tabla 1. Variables independientes sectores económicos

\begin{tabular}{|l|l|l|}
\hline \multicolumn{1}{|c|}{ Sector primario } & Sector secundario & Sector terciario \\
\hline $\begin{array}{l}\text { Promedio detallado } \\
\text { de precipitaciones } \\
\text { (mm anuales) }\end{array}$ & $\begin{array}{l}\text { Promedio detallado } \\
\text { de precipitaciones } \\
\text { (mm anuales) }\end{array}$ & $\begin{array}{l}\text { Promedio } \\
\text { detallado de } \\
\text { precipitaciones } \\
\text { (mm anuales) }\end{array}$ \\
\hline $\begin{array}{l}\text { El porcentaje de } \\
\text { gastos efectuados a } \\
\text { nivel mundial }\end{array}$ & $\begin{array}{l}\text { Comercio de mer- } \\
\text { caderías del sector }\end{array}$ & $\begin{array}{l}\text { Comercio de } \\
\text { mercaderías del } \\
\text { sector }\end{array}$ \\
\hline $\begin{array}{l}\text { Índice de produc- } \\
\text { ción animal }\end{array}$ & $\begin{array}{l}\text { Costo de los facto- } \\
\text { res de producción }\end{array}$ & $\begin{array}{l}\text { Índice del gasto } \\
\text { de consumo final }\end{array}$ \\
\hline $\begin{array}{l}\text { Índice de produc- } \\
\text { ción de alimentos }\end{array}$ & $\begin{array}{l}\text { Rentas totales de } \\
\text { los recursos para la } \\
\text { producción }\end{array}$ & $\begin{array}{l}\text { Índice de con- } \\
\text { sumo final }\end{array}$ \\
\hline $\begin{array}{l}\text { Inflación, precios al } \\
\text { consumidor }\end{array}$ & & \\
\hline
\end{tabular}

Se utilizó el programa estadístico EViews 7 de la paquetería Microsoft Windows, usado para análisis econométrico, con el fin de ejecutar la regresión múltiple tomando en cuenta las variables explicadas con anterioridad. En esta regresión, se analizan ciertas pruebas estadísticas con el fin de asegurar la certeza del modelo respecto de la estimación de las variables. A continuación, se presentan las pruebas:

- $R^{2}$ significativa, es decir, que la correlación entre las variables sea cercana a 1

- Prueba de multicolinealidad con valores de parámetros significativos, es decir, menores a 0,05\%

- Prueba de normalidad de Jarque-Bera (mayor a la distribución $X^{2}$ con 2 grados de libertad (valor 
en tablas de $\left.X^{2}, 5,99\right)$ y valor $\mathrm{p}$ significativa, es decir, menores a $0,05 \%$

- Prueba de normalidad de Anderson-Darling (mayor a valor 0,05 )

- Prueba de normalidad de Kolmogorov (mayor a valor p 0,05)

- Prueba de heteroscedasticidad de White con datos cruzados y no cruzados (mayor a valor p 005\% y prueba distribución F significativa)

- Prueba de autocorrelación con parámetros significativos a las bandas de confianza

- Prueba de estabilidad, mediante la prueba de cuadrados CUSUM, donde el modelo no debe sobrepasar las bandas de confianza

Si el modelo econométrico logra pasar las pruebas mencionadas con anterioridad, se entenderá que los coeficientes de la ecuación de la regresión indican un porcentaje de explicación respecto de la variable dependiente, es decir, la explicación en la producción de los sectores económicos. Por lo tanto, la regresión indicará el porcentaje de sensibilidad de la producción respecto del promedio de precipitación y las demás variables tomadas en cuenta.

\subsection{Exposición y sensibilidad del impacto del riesgo climático en operaciones de las líneas aéreas}

En esta sección, se analiza el impacto que tiene el comportamiento del clima (en este caso, la precipitación) en las operaciones de las líneas aéreas. Se seleccionó este sector, debido a que se puede apreciar directamente el impacto del clima en sus operaciones, ya que se realizan directamente en el aire.
Para este análisis, también se realizó una regresión múltiple con la misma paquetería EViews 7; se tomó como variable dependiente el transporte aéreo de partidas de vuelos en todo el mundo de compañías. A continuación, en la tabla 2 , se describen las variables independientes utilizadas con el fin de medir la sensibilidad y buscar la corrección existente entre las variables tomadas. Todos los datos fueron obtenidos de la base datos del Banco Mundial.

\section{Tabla 2. Variables independientes sector aéreo}

\begin{tabular}{|l|}
\hline Promedio detallado de precipitaciones (mm anuales) \\
\hline Comercio de mercaderías (\% del PIB) \\
\hline Gasto del sector \\
\hline Índice de consumo final \\
\hline
\end{tabular}

De la misma manera que los modelos econométricos anteriores, este modelo tiene que pasar las mismas pruebas $\left(R^{2}\right.$ significativa, prueba de multicolinealidad, prueba de normalidad de Jarque-Bera, prueba de normalidad de Anderson-Darling, prueba de heteroscedasticidad de White, prueba de autocorrelación y prueba de estabilidad), ya que, si el modelo es eficiente, se podrá probar la relación o explicación que guardan las variables entre sí.

\subsubsection{Capacidad adaptativa}

En esta sección, se analiza la posibilidad de implementar medidas adaptativas, que van de la mano con el análisis de vulnerabilidad que se detectó en la sección anterior. El diseño de medidas de adaptación, como se mencionó en la primera parte de esta investigación, sirve para minimizar el impacto negativo que tiene el comportamiento del clima en las actividades económicas. Su diseño se basa en la sensibilidad que tiene cada sector en específico, es decir, las medidas se implementan de acuerdo con las necesidades de cada actividad. 


\section{Resultados}

En esta sección, se describe y se analiza el impacto que tiene el comportamiento del clima en las actividades económicas en cada sector en particular. A continuación, se presentan los resultados obtenidos en cuanto a la elaboración de la metodología descrita anteriormente.

\subsection{Descripciones de las vulnerabilidades de cada sector}

El comportamiento del clima ha presentado una tendencia hacia el alza debido a las repercusiones que tiene el cambio climático en el planeta. De acuerdo con la Comisión Económica para América Latina y el Caribe (Cepal, 2017), los desastres naturales meteorológicos han presentado un incremento en los últimos cincuenta años, seguido por desastres hidrológicos. Así mismo, de acuerdo con Cepal, en los últimos quince años, los desastres naturales se han agravado respecto de los cincuenta ańos anteriores como consecuencia del cambio climático. Ya que los desastres naturales han sido más frecuentes en los últimos años, se han generado mayores pérdidas en la economía. Como las actividades pertenecientes a los sectores económicos se vuelven más sensibles, se hace necesario el desarrollo de medidas adaptativas que fortalezcan la economía ante la incertidumbre generada por el cambio anómalo en el clima.

Como se ha comentado, cada sector económico es importante para la sociedad, ya que, sin los recursos que cada uno de ellos genera, no sería posible la subsistencia del sistema. Es de vital importancia reconocer las vulnerabilidades que tiene cada sector, pues, de no hacerlo, estaría expuesto a los cambios anómalos del clima, y, por lo tanto, a la incertidumbre sobre la producción y rendimiento. A continuación, se presenta la descripción de las vulnerabilidades de cada sector:

\section{Sector primario}

El sector primario es fundamental para el sostenimiento de la población humana en dos aspectos principales: en primer lugar, provee a la población humana de alimentos, materias primas, medicinas y otros bienes económicos; en segundo lugar, provee de servicios ecosistémicos, como cultura y tradiciones en relación con la actividad agrícola, biodiversidad, formación de suelo, y regulación de los ciclos hídricos, entre otros. La vulnerabilidad que tiene el comportamiento del clima (cambio climático) en el sector primario se presenta en la distribución de la vegetación, ya que influye directamente en la producción de los cultivos al afectar directamente la calidad y el volumen de la producción. Esto genera incertidumbre acerca de la capacidad o rendimiento del sistema productivo para funcionar eficientemente.

El aumento en la temperatura provocado por el cambio climático tiene impactos positivos al inicio tanto en la producción como en los rendimientos del sector primario (ganadería, agricultura, pesca, etc.). Sin embargo, a medida que sigue incrementando el nivel de temperatura, el impacto tiende a ser negativo, ya que los procesos naturales se vuelven inestables, el agua para el riesgo de cultivos se vuelve escasa, etcétera. Además, el aumento de la temperatura conlleva al cambio en los patrones de precipitación debido a los ciclos bioquímicos que presenta el. Esto implica un mayor nivel de incertidumbre en el comportamiento del clima, aunado a las proyecciones que pueden realizarse sobre este.

En resumen, se prevén efectos diferenciados y divergentes en relación a los patrones esperados de temperatura y lluvias. En ciertos rangos, dichos efectos pueden incrementar el rendimiento de los cultivos de temporal. Sin embargo, la incertidumbre y el riesgo de salirse 
de esos rangos en los patrones traerían consigo una reducción importante de los rendimientos del sector primario, como las horas activas de pastoreo y mayor incidencia en procesos infecciosos en el ganado, lo que provoca desequilibrios en dichos ciclos. Aun así, un aspecto positivo a señalar es la reducción de costes destinados a la protección del ganado durante los inviernos, ya que las temperaturas se suavizarán, aunque los aumentos de temperatura media son significativamente mayores en los meses de verano que en los de invierno.

\section{Sector secundario}

El sector secundario brinda principalmente producción y distribución industrial a la población y a los ecosistemas. Por ello, el cambio climático tiene efectos positivos y negativos para este sector. Uno de los efectos del cambio climático es que aumenta la temperatura del planeta, por lo que los polos tienden a derretirse y, en consecuencia, el nivel del mar asciende. Dicho aumento pone en desventaja a las empresas industriales debido a su ubicación, ya que, si se encuentran en la costa, la producción quedará vulnerable ante el comportamiento del nivel del mar, pues es donde el ascenso del nivel del mar es más marcado. Por lo tanto, las instalaciones industriales o de generación de energía localizadas en la costa serán más vulnerables. También lo serán las terminales de abastecimiento y distribución de hidrocarburos y sus derivados, y la infraestructura de conexión (carreteras, vías férreas) en la misma ubicación. En consecuencia, la vulnerabilidad de la industria y los sistemas energéticos se encuentra en función de las necesidades de abastecimiento de determinados volúmenes de agua, de la regularidad con que se requieren de dichos abastos y del clima del lugar en el que se ubica la empresa.
Por lo tanto, la mayor vulnerabilidad que tiene el sector secundario está presente en la obtención de recursos para la producción, ya que derivan directamente de los ecosistemas. Por ello, la mayor afectación que presenta este sector es el precio del productor. De igual forma, empresas industriales y generadoras de energía quedan expuestas al cambio en el comportamiento de las precipitaciones, ya que pueden generar sequías e inundaciones. En el caso de empresas con sedes en zonas secas, la principal vulnerabilidad se observará en la obtención de recursos hídricos debido a la sequía por el aumento de la temperatura; cuando el agua comience a escasear, será necesario invertir más para mantener el rendimiento y producción deseados, lo que implicaría un costo adicional al establecido. Lo mismo aplica para la obtención de energía eléctrica, y en el de extracción y refinación de combustibles fósiles.

\section{Sector terciario}

El sector terciario es el sector en el que se prestan servicios y se intercambian productos terminados, entre otras actividades similares. Las vulnerabilidades que presenta este sector implican las vulnerabilidades de los dos primeros sectores, ya que, para poder obtener el producto terminado, se tiene que producir en cualquiera de los dos primeros sectores. Por ello, el sector terciario es aún más sensible a los cambios en el comportamiento del clima.

Además de las vulnerabilidades del sector primario y del secundario, el sector terciario enfrenta la posibilidad de daño en relación a la venta de productos terminado, ya sea por fenómenos naturales, o cambios drásticos en la precipitación y en el nivel de temperatura. La sede de venta y la demanda del consumidor se pueden ver perjudicadas, ya que las ganancias dependen del comportamiento del cliente. Por ejemplo, 
en el caso de venta de calcetas, si durante un año el invierno es más cálido de lo esperado, las ventas de este producto serán menores con respecto a años anteriores, lo que afectaría la certidumbre de las ganancias. En el caso de servicios, es decir, productos intangibles, se puede tomar como ejemplo el caso del turismo. El clima afecta directamente los rendimientos que puede obtener este sector, ya que las ganancias dependen de la demanda de consumidores, que depende del comportamiento del clima. Además, el sector transporte también se ve afectado por el comportamiento del consumidor y del clima: existe una relación negativa entre estas partes, tanto en la construcción de infraestructuras y en la planificación de obras, como en el transporte de mercancías o personas.

El sector de las aseguradoras puede ser uno de los que más rápida e intensamente se puede ver afectado por los cambios climáticos. Por ejemplo, las empresas turísticas y agrícolas tendrán que recurrir a seguros especiales para situaciones climáticas extremas. Además, otro efecto del cambio climático ha sido el incremento del número y valor de los bienes asegurados. La evolución del clima puede agudizar estas tendencias, lo que provocaría un aumento de las primas contratadas.

\subsubsection{Ejemplos sobre el impacto en sectores económicos}

Las situaciones de riesgo climático pueden incidir en empresas de muchos sectores: desde empresas manufactureras y agrarias, hasta líneas aéreas. Por ejemplo, para una empresa del sector energético se vuelve primordial el comportamiento del clima debido a la demanda presentada por el servicio; es decir, los cambios climáticos no previstos afectan las expectativas de oferta y demanda del servicio energético, lo que trae como consecuencia encarecimiento y/o abaratamiento tanto del costo de insumos o suministros de producción como del bien o servicio que se comercializa. Tomando el ejemplo de la empresa energética, a continuación, se ilustran algunos casos en los que se identifica el impacto en el cambio de comportamiento del clima.

- Invierno más frío respecto de años anteriores:

En este caso, la demanda tenderá a incrementar debido a la necesidad de obtener energía para calefacción, ya sea a través de electricidad, de gas, etc. Por un lado, para la empresa energética esta situación implicará el aumento de los precios de su servicio, siempre y cuando se encuentren en un mercado no competitivo, además del aumento en el costo de producción. Por otro lado, ya que la empresa se encuentra en un mercado competitivo, incurrirá en pérdidas debido a que tendrá que absorber el aumento de los costos productivos. El aumento no previsto en la demanda puede sobrepasar la capacidad instalada de la empresa para prestar el servicio; en este caso, resultarían cortes en el suministro del servicio, lo cual afectaría la rentabilidad de la empresa y al consumidor de dichos servicios.

- Invierno más cálido respecto de años anteriores:

En este caso, la demanda tenderá a bajar debido al buen clima. Esto provocará que haya exceso de energía en el almacén de la empresa; es decir, debido al cambio en el clima, el stock que la empresa tenía previsto para esta temporada será mayor al que realmente demandarán sus clientes, por lo que se generarán pérdidas para la empresa. Como se puede inferir, existe una alta correlación entre el cambio en el clima (en la temperatura), y los cambios en la oferta y la demanda de energía. Por ello, se identifica el riesgo potencial en las operaciones de las empresas, así como la necesidad de cobertura para las empresas. 


\subsection{Exposición y sensibilidad del impacto al riesgo climático}

Esta sección se enfoca en el análisis de sensibilidad del impacto y exposición, concretamente en los cambios en la producción de la variable macroeconómica PIB de cada sector económico con respecto al comportamiento del cambio del clima. Dicho análisis tiene el fin de dimensionar la producción que podrán tener o no las empresas de los diferentes sectores por cada punto de desviación frente al promedio de precipitación mundial anual, midiendo la correlación que existe entre ambos factores con el fin de determinar el grado de vulnerabilidad que existe.

Se determinaron regresiones lineales múltiples con el fin de estimar el comportamiento de la producción efectuada por cada sector económico respecto de cambios en el comportamiento del promedio de precipitación anual determinado por el Banco Mundial. La regresión múltiple toma en cuenta como variable dependiente el PIB de cada sector en particular expresado en variaciones porcentuales anuales. Las variables independientes están relacionadas con cada sector en particular. Para el sector primario se tomó el índice de producción animal, el índice de cosecha, el porcentaje de gastos efectuados a nivel mundial y el índice de producción de alimentos. Para el sector secundario se utilizó el valor agregado del sector industrial, el consumo de mercancías, el costo de factores de la producción, las rentas de recursos para la producción, el consumo de energía eléctrica y la inflación. Por último, para el sector terciario se utilizó el comercio de servicios, el índice de gasto de consumo final, el valor agregado del sector de servicios, el índice de consumo final y el gasto para el consumo final.

Con ayuda del programa estadístico EViews-7, se ejecutó el cálculo de una regresión lineal múltiple tomando en cuenta las variables explicadas con anterioridad con el fin de determinar la relación que guardan las variables respecto de la producción que tiene cada sector económico. Los resultados se aprecian en las figuras 3, 4 y 5 , en las que se resalta el periodo de análisis y el método de mínimos cuadrados utilizado.

La ecuación resultante para la regresión en el caso del mercado español fue la siguiente:

$$
Y_{i}=\beta_{1}+\beta_{2}+\beta_{3}+\beta_{4}+\beta_{5}
$$

Donde:

$Y_{i}=$ Demanda pasajeros Aeropuerto Madrid-Barajas (DEMANDA MADRID)

$\beta_{1}=$ PIB industrial (índice) trimestral (PIB)

$\beta_{2}=$ Precipitación España trimestral (lluvia)

$\beta_{3}=$ Pasajeros transportados trimestral (INE)

$\beta_{4}=$ Índices de precios del sector servicios por sectores (ÍNDICE PSS)

$\beta_{5}=$ Índice precios al productor (IPP)

Figura 3. Regresión múltiple sector primario

Dependent Variable: PIBprimario

Method: Least Squares

Date: 03/29/19 Time: 17:47

Sample (adjusted): 20012017

Included observations: 17 after adjustments

\begin{tabular}{crrrc}
\hline \hline Variable & Coefficient & Std. Error & t-Statistic & Prob. \\
\hline \hline C & 37.07246 & 3.794309 & 9.770541 & 0.0000 \\
LLUVIA & -0.010174 & 0.002109 & -4.823171 & 0.0005
\end{tabular}

*Regresión obtenida a través del software EViews-7

La ecuación resultante para la regresión en el caso del mercado español fue la siguiente:

$$
Y_{i}=\beta_{1}+\beta_{2}+\beta_{3}+\beta_{4}+\beta_{5}
$$

Donde:

$Y_{i}=$ Demanda pasajeros Aeropuerto Madrid-Barajas (DEMANDA MADRID)

$\beta_{1}=$ PIB industrial (índice) trimestral (PIB)

$\beta_{2}=$ Precipitación España trimestral (lluvia) 
$\beta_{3}=$ Pasajeros transportados trimestral (INE)

$\beta_{4}=$ Índices de precios del sector servicios por sectores (ÍNDICE PSS)

$\beta_{5}=$ Índice precios al productor (IPP)

Figura 4. Regresión múltiple sector secundario

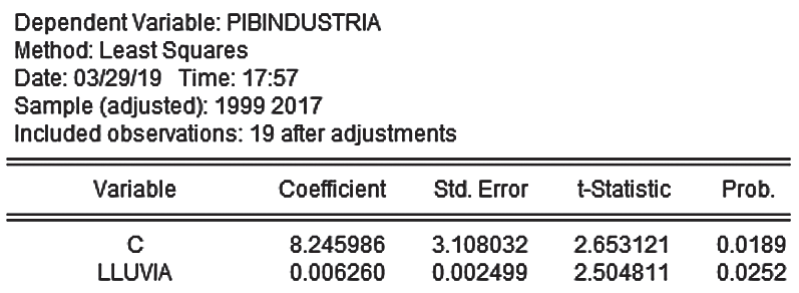

*Regresión obtenida a través del software EViews-7

La ecuación resultante para la regresión en el caso del mercado español fue la siguiente:

$$
Y_{i}=\beta_{1}+\beta_{2}+\beta_{3}+\beta_{4}+\beta_{5}
$$

Donde:

$Y_{i}=$ Demanda pasajeros Aeropuerto Madrid-Barajas (DEMANDA MADRID)

$\beta_{1}=$ PIB industrial (índice) trimestral (PIB)

$\beta_{2}=$ Precipitación Espańa trimestral (lluvia)

$\beta_{3}=$ Pasajeros transportados trimestral (INE)

$\beta_{4}=$ Índices de precios del sector servicios por sectores (ÍNDICE PSS)

$\beta_{5}=$ Índice precios al productor (IPP)

\section{Figura 5. Regresión múltiple sector terciario}

Dependent Variable: PIBSERVICIOS

Method: Least Squares

Date: 04/01/19 Time: 09:45

Sample (adjusted): 19992017

Included observations: 19 after adjustments

\begin{tabular}{crrrr}
\hline \hline Variable & Coefficient & Std. Error & t-Statistic & Prob. \\
\hline \hline C & 50.87621 & 9.597871 & 5.300781 & 0.0001 \\
LLUVIA(-2) & -0.012554 & 0.003871 & -3.243510 & 0.0059
\end{tabular}

*Regresión obtenida a través del software EViews-7

Los modelos econométricos obtuvieron las siguientes pruebas:
Tabla 3. Resultados de los modelos econométricos aplicados

\begin{tabular}{|l|l|}
\hline \multicolumn{1}{|c|}{ Prueba } & \multicolumn{1}{c|}{ Interpretación } \\
\hline Multicolinealidad & $\begin{array}{l}\text { Valor menor a 0,05, lo cual rati- } \\
\text { fica que el modelo es eficiente }\end{array}$ \\
\hline $\begin{array}{l}\text { Normalidad de } \\
\text { Jarque-Bera }\end{array}$ & $\begin{array}{l}\text { Valor mayor a la distribución } X^{2}, \\
\text { con } 2 \text { grados de libertad (valor } \\
\left.\text { en tablas de } X^{2}, 5,99\right) \text { y valor p } \\
\text { significativa, es decir, menores a } \\
0,05 \%\end{array}$ \\
\hline $\begin{array}{l}\text { Prueba de normalidad de } \\
\text { Anderson-Darling }\end{array}$ & $\begin{array}{l}\text { Valor p mayor a 0,05, es decir, es } \\
\text { significativo }\end{array}$ \\
\hline $\begin{array}{l}\text { Prueba de normalidad de } \\
\text { Kolmogorov }\end{array}$ & $\begin{array}{l}\text { Valor p mayor a 0,05, es decir, es } \\
\text { significativo. }\end{array}$ \\
\hline $\begin{array}{l}\text { Prueba de heteroscedasti- } \\
\text { cidad de White con datos } \\
\text { cruzados y no cruzados }\end{array}$ & $\begin{array}{l}\text { Valor p mayor a 0,05\% y prueba } \\
\text { distribución F significativa, es } \\
\text { decir, el modelo es eficiente. }\end{array}$ \\
\hline Prueba de autocorrelación & $\begin{array}{l}\text { Parámetros significativos dentro } \\
\text { de las bandas de confianza }\end{array}$ \\
\hline $\begin{array}{l}\text { Prueba de estabilidad, } \\
\text { mediante la prueba de } \\
\text { cuadrados CUSUM }\end{array}$ & $\begin{array}{l}\text { Las variables no sobrepasan las } \\
\text { bandas de confianza, lo que da } \\
\text { estabilidad a la estimación de } \\
\text { variables. }\end{array}$ \\
\hline $\begin{array}{l}R^{2} \text { (Correlación entre } \\
\text { variables) }\end{array}$ & $\begin{array}{l}\text { Existe una relación positiva entre } \\
\text { las variables, por lo que esta } \\
\text { prueba es significativa. }\end{array}$ \\
\hline & \\
\hline
\end{tabular}

Las pruebas efectuadas en los modelos econométricos revelan que las variables independientes no muestran dependencia entre sí; además, tienen correlación positiva y alta, y estabilidad en las variables. Esto demuestra que los modelos son confiables para la estimación de las variables y su relación entre ellas.

El análisis de sensibilidad sobre datos históricos arroja los coeficientes de las ecuaciones de las regresiones e indica el porcentaje de explicación respecto de la variable dependiente, es decir, la explicación en la producción de cada sector económico. Por lo tanto, las regresiones indican el porcentaje de sensibilidad (cambio) de la producción de cada sector económico respecto del promedio de precipitación y las demás 
variables analizadas en cada sector económico. En síntesis, se manifiesta que, por cada aumento de un punto en el promedio de precipitación, la producción presentada por el sector primario, secundario y terciario cambia un $10,17 \%, 6,2 \%$ y $12,55 \%$ para el sector primario, secundario y terciario, respectivamente.

\subsection{Análisis de sensibilidad del impacto y exposición en aerolíneas}

En esta sección, se analiza el impacto que tiene el comportamiento del clima, concretamente, la precipitación a nivel global en las operaciones de las líneas aéreas ante los cambios en el transporte aéreo histórico. Dicho análisis tiene el fin de dimensionar la cantidad de vuelos que podrá haber por cada punto de desviación frente al promedio de precipitación mundial anual, midiendo la correlación que existe entre ambos factores con el fin de determinar el grado de vulnerabilidad existente.

De igual forma, se determinó una regresión lineal múltiple a través del programa EViews con el fin de estimar el comportamiento de los vuelos con respecto a cambios en el comportamiento del promedio de precipitación anual determinado por el Banco Mundial. La regresión múltiple toma en cuenta como variable dependiente el transporte aéreo (vuelos ocurridos) expresado en unidades anuales. Como variables independientes, se analizaron el promedio detallado de precipitaciones, PIB del sector, índice de consumo final y el gasto del sector. Los resultados de la regresión se aprecian en la figura 6 , en la que se resalta el periodo de análisis y el método de mínimos cuadrados utilizado.

La ecuación resultante para la regresión en el caso del mercado español fue la siguiente:

$$
Y_{i}=\beta_{1}+\beta_{2}+\beta_{3}+\beta_{4}+\beta_{5}
$$

Donde:

$Y_{i}=$ Transporte aéreo, partidas de vuelos en todo el mundo de compañías

$\beta_{1}=$ Comercio de mercaderías (\% del PIB)

$\beta_{2}=$ Precipitación España trimestral (lluvia)

$\beta_{3}=$ Índice de consumo final

$\beta_{4}=$ Gastos del sector

Figura 6. Regresión múlltiple sector aéreo

Dependent Variable: LOG(TAEREO)

Method: Least Squares

Date: 04/22/19 Time: 16:18

Sample (adjusted): 20012017

Included observations: 17 after adjustments

\begin{tabular}{ccccc}
\hline \hline Variable & Coefficient & Std. Error & t-Statistic & Prob. \\
\hline \hline C & 14.44045 & 0.948607 & 15.22279 & 0.0000 \\
LLUVIA(-2) & 0.000835 & 0.000343 & 2.431751 & 0.0316
\end{tabular}

*Regresión obtenida a través del software EViews-7

De la misma manera que los modelos econométricos anteriores, los resultados presentados en este modelo pasaron las pruebas requeridas para concluir que son eficientes. Las pruebas son $\mathrm{R}^{2}$ significativa, prueba de multicolinealidad, prueba de normalidad de JarqueBera, prueba de normalidad de Anderson-Darling, prueba de heteroscedasticidad de White, prueba de autocorrelación y prueba de estabilidad. Por lo tanto, el modelo prueba la relación o explicación que guardan las variables entre sí. Además, las pruebas efectuadas en los modelos econométricos revelan que las variables independientes no muestran dependencia entre sí, y tienen correlación positiva y alta. Además, tienen estabilidad en las variables, lo que demuestra la eficiencia de los modelos para la estimación de las variables y su relación entre ellas.

El análisis de sensibilidad sobre datos históricos en el sector de las líneas aéreas arroja los coeficientes de las ecuaciones de las regresiones e indica el porcentaje de explicación respecto de la variable dependiente, es decir, la explicación en el transporte aéreo. Por lo tanto, 
las regresiones indican el porcentaje de sensibilidad del transporte aéreo respecto del promedio de precipitación y las demás variables analizadas. En síntesis, se manifiesta que, por cada aumento de un punto en el promedio de precipitación, el transporte aéreo cambia un $0,835 \%$.

\subsubsection{Capacidad adaptativa}

Los resultados arrojados por los modelos econométricos determinan la relación existente entre factores meteorológicos (precipitación) y la producción de los sectores económicos, lo que implica el impacto tanto positivo como negativo para cada cambio que presente el clima en cada región, lo que vuelve sensibles (vulnerables) las actividades económicas. Una vez demostrada la vulnerabilidad que tienen las actividades económicas, es necesario implementar medidas enfocadas para enfrentar los impactos negativos del clima. Dichas medidas adaptativas deben asegurar la eficiente producción de cada sector económico, ya sea a través de seguros climáticos o de derivados financieros cuyo subyacente sea el comportamiento del clima. La implementación de estas medidas depende de la zona geográfica, y del avance económico y tecnológico que se tenga en la región.

\section{Conclusiones}

El cambio en el comportamiento del clima ha creado la necesidad de anticiparse ante los desastres que puede generar dicho cambio. Por su parte, las empresas requieren de la identificación y adaptación ante este comportamiento anómalo, ya que las consecuencias del cambio climático podrían ser negativas para sus ganancias.

En esta investigación, se analizó el impacto que tiene el comportamiento anómalo del clima en los dife- rentes sectores económicos (primario, secundario y terciario). Se resaltó que todas las actividades tienen una gran sensibilidad ante el clima debido a la interrelación que presentan las actividades productivas entre sí. De esta forma, es necesaria la adaptación ante los cambios climáticos con el fin de poder minimizar la vulnerabilidad de las actividades, ya que en el largo plazo el impacto será mayor.

Por esta razón, se vuelve necesario implementar medidas correctivas o adaptativas (estrategias de administración del riesgo) que ayuden a identificar la correlación que existe entre las actividades económicas con el comportamiento del clima. Esto tiene el fin de facilitar o mejorar la capacidad de enfrentar los riesgos climáticos, pues, mientras mejor se identifique la vulnerabilidad ante el cambio climático, mejor certidumbre tendrán los rendimientos de las empresas.

\section{Referencias bibliográficas}

Comisión Económica para América Latina y el Caribe (Cepal). (abril, 2017). Boletín Desafíos. Niñez y desastres en América Latina y El Caribe, (20).

Conde, C., Ferrer, R., Gay, C. \& Araujo, R. (2004). Impactos del cambio climático en la agricultura en México. En J. Martínez \& A. Fernández (Comps.), Cambio climático: una visión desde México (pp. 227-238). México: SEMARNAT-INE.

Duarte Cueva, F. (2014). Efectos del cambio climático en la economía, el comercio internacional y la estrategia empresarial. Contabilidad y Negocios, 9(18), 75-98.

Hong, H., Weikai, F. \& Jiangmin, X. (September 7, 2017). Climate Risks and Market Efficiency. Journal of Econometrics, Forthcoming. https://doi.org/10.2139/ ssrn. 2776962

Hsiang, S. M. (2016). Climate Econometrics (NBRE Working Paper 22181). Cambridge: National Bureau of Economic Research. https://doi.org/10.3386/w22181

Contabilidad y Negocios (15) 29, 2020 / ISSN 1992-1896 
Instituto Nacional de Ecología y Cambio Climático de México (2020). Portal del Instituto Nacional de Ecología y Cambio Climático de México. Recuperado de https://www.gob.mx/inecc

Monjas, M. (2010). Derivados meteorológicos: una alternativa de cobertura de riesgos para la empresa. Boletín de Estudios Económicos, LXV(199), 19-37.

O’Brien, K. \& Leichenko, R. (2000). Double Exposure: Assessing the Impacts of Climate Change Within the Context of Economic Globalization. Global Environmental Change, 10(3), 221-232. https://doi. org/10.1016/S0959-3780(00)00021-2

Ortiz-Paniagua, C.F. \& Pérez, F. B. (2017). Migración, deterioro ambiental y cambio climático: hacia un modelo bajo la perspectiva del análisis regional. Acta Universitaria, 27(1), 46-58 https://doi.org/10.15174/ au.2017.1474

Schlenker, W. \& Taylor, C. A. (2019). Market Expectations About Climate Change (NBER Working Paper 25554). Cambridge: National Bureau of Economic Research. https://doi.org/10.3386/w25554

Smit, B., Ford, J. \& Wandel, J. (2006). Vulnerability to Climate change in the Arctic: A Case Study from Arctic Bay, Canada. Science Direct, 16(2), 145-160. https:// doi.org/10.1016/j.gloenvcha.2005.11.007

Štulec, I. (2017). Effectiveness of Weather Derivatives as a Risk Management Tool in Food Retail: The Case of
Croatia. International Journal of Financial Studies, (5), 0-15. https://doi.org/10.3390/ijfs5010002

United Nations Framework Convention on Climate Change (UNFCCC). (11 de diciembre, 2007). Programa de trabajo de Nairobi sobre los efectos, la vulnerabilidad y la adaptación al cambio climático. Recuperado de https://unfccc.int/resource/docs/2007/sbsta/spa/ 117 s.pdf

Villalba Fonfría, A. (agosto, 2008). Panorámica de la problemática medioambiental en el mundo y en España. CIRIEC-España, Revista de Economía Pública, Social y Cooperativa, (61), 7-30.

Wang, S. L., Ball, E., Nehring, E., Williams, R. \& Chau, T. (2017). Impacts of Climate Change and Extreme Weather on U.S. Agricultural Productivity: Evidence and Projection (NBER Working Paper 23533). Cambridge, MA: National Bureau of Economic Research. https://doi.org/10.3386/w23533

Whalley, J. \& Yuan, Y. (2009). Global Financial Structure and Climate Change (NBER Working Paper 14888). Cambridge: National Bureau of Economic Research. https://doi.org/10.3386/w14888

Fecha de recepción: 29 de noviembre de 2019 Fecha de aceptación: 12 de febrero de 2020 Correspondencia: ilopez@ccee.ucm.es rotinb@comunidad.unam.mx 


\section{Anexos}

\section{Anexo 1. Resultados regresión múltiple sector primario}

Dependent Variable: PIBprimario

Method: Least Squares

Date: 03/29/19 Time: 17:47

Sample (adjusted): 20012017

Included observations: 17 after adjustments

\begin{tabular}{lrrrr}
\hline \hline \multicolumn{1}{c}{ Variable } & Coefficient & Std. Error & t-Statistic & Prob. \\
\hline \hline C & 37.07246 & 3.794309 & 9.770541 & 0.0000 \\
LLUVIA & -0.010174 & 0.002109 & -4.823171 & 0.0005 \\
INFLAC(-3) & 0.207408 & 0.052304 & 3.965459 & 0.0022 \\
PRODUALIMEN(-4) & 0.171034 & 0.035612 & 4.802720 & 0.0006 \\
PRODUANIM(-1) & -0.236280 & 0.030097 & -7.850674 & 0.0000 \\
$\quad$ GTO(-2) & -0.233832 & 0.095786 & -2.441192 & 0.0328 \\
\hline \hline R-squared & 0.964095 & Mean dependent var & 13.52370 \\
Adjusted R-squared & 0.947774 & S.D. dependent var & 0.868519 \\
S.E. of regression & 0.198482 & Akaike info criterion & -0.125673 \\
Sum squared resid & 0.433346 & Schwarz criterion & 0.168402 \\
Log likelihood & 7.068221 & Hannan-Quinn criter. & -0.096441 \\
F-statistic & 59.07271 & Durbin-Watson stat & 1.753063 \\
Prob(F-statistic) & 0.000000 & & & \\
\hline \hline
\end{tabular}

*Regresión obtenida a través del software EViews-7

\section{Anexo 2. Resultados regresión múltiple sector secundario}

Dependent Variable: PIBINDUSTRIA

Method: Least Squares

Date: 03/29/19 Time: 17:57

Sample (adjusted): 19992017

Included observations: 19 after adjustments

\begin{tabular}{lrlll}
\hline \hline \multicolumn{1}{c}{ Variable } & Coefficient & Std. Error & t-Statistic & Prob. \\
\hline \hline C & 8.245986 & 3.108032 & 2.653121 & 0.0189 \\
LLUVIA & 0.006260 & 0.002499 & 2.504811 & 0.0252 \\
COMERCIOM & 0.226249 & 0.020395 & 11.09354 & 0.0000 \\
CTOFACTOR(-2) & $-2.67 \mathrm{E}-12$ & $6.19 \mathrm{E}-13$ & -4.311508 & 0.0007 \\
RENTARECU(-1) & 0.109855 & 0.056321 & 1.950525 & 0.0714 \\
\hline \hline R-squared & 0.960255 & Mean dependent var & 28.77447 \\
Adjusted R-squared & 0.948900 & S.D. dependent var & 1.293271 \\
S.E. of regression & 0.292349 & Akaike info criterion & 0.599199 \\
Sum squared resid & 1.196554 & Schwarz criterion & 0.847736 \\
Log likelihood & -0.692394 & Hannan-Quinn criter. & 0.641262 \\
F-statistic & 84.56178 & Durbin-Watson stat & 1.981583 \\
Prob(F-statistic) & 0.000000 & & & \\
\hline \hline
\end{tabular}

*Regresión obtenida a través del software EViews-7

\section{Anexo 3. Resultados regresión múltiple sector terciario}

Dependent Variable: PIBSERVICIOS

Method: Least Squares

Date: 04/01/19 Time: 09:45

Sample (adjusted): 19992017

Included observations: 19 after adjustments

\begin{tabular}{lrlrr}
\hline \hline \multicolumn{1}{c}{ Variable } & Coefficient & Std. Error & t-Statistic & Prob. \\
\hline \hline C & 50.87621 & 9.597871 & 5.300781 & 0.0001 \\
LLUVIA(-2) & -0.012554 & 0.003871 & -3.243510 & 0.0059 \\
COMERCIOM(-1) & 0.073137 & 0.023905 & 3.059423 & 0.0085 \\
GTOCONSFIN(-1) & $1.54 \mathrm{E}-14$ & $3.22 \mathrm{E}-15$ & 4.781361 & 0.0003 \\
CONSUMFINAL & -0.257508 & 0.088961 & -2.894629 & 0.0118 \\
\hline \hline R-squared & 0.959255 & Mean dependent var & 21.02282 \\
Adjusted R-squared & 0.947614 & S.D. dependent var & 1.513896 \\
S.E. of regression & 0.346500 & Akaike info criterion & 0.939069 \\
Sum squared resid & 1.680876 & Schwarz criterion & 1.187605 \\
Log likelihood & -3.921153 & Hannan-Quinn criter. & 0.981131 \\
F-statistic & 82.40074 & Durbin-Watson stat & 1.756042 \\
Prob(F-statistic) & 0.000000 & & & \\
\hline \hline
\end{tabular}

*Regresión obtenida a través del software EViews-7

\section{Anexo 4. Resultados regresión múltiple sector aéreo}

Dependent Variable: LOG(TAEREO)

Method: Least Squares

Date: 04/22/19 Time: $16: 18$

Sample (adjusted): 20012017

Included observations: 17 after adjustments

\begin{tabular}{lrrrr}
\hline \hline \multicolumn{1}{c}{ Variable } & Coefficient & Std. Error & t-Statistic & Prob. \\
\hline \hline C & 14.44045 & 0.948607 & 15.22279 & 0.0000 \\
LLUVIA(-2) & 0.000835 & 0.000343 & 2.431751 & 0.0316 \\
PIB(-4) & 0.008518 & 0.002755 & 3.092379 & 0.0093 \\
CONSUMOFIN & -0.027904 & 0.008587 & -3.249720 & 0.0070 \\
GTOSECTOR(-1) & $3.80 \mathrm{E}-15$ & $3.40 \mathrm{E}-16$ & 11.16295 & 0.0000 \\
\hline \hline R-squared & 0.980957 & Mean dependent var & 14.09808 \\
Adjusted R-squared & 0.974610 & S.D. dependent var & 0.207369 \\
S.E. of regression & 0.033043 & Akaike info criterion & -3.742101 \\
Sum squared resid & 0.013102 & Schwar criterion & -3.497038 \\
Log likelihood & 36.80786 & Hannan-Quinn criter. & -3.717741 \\
F-statistic & 154.5416 & Durbin-Watson stat & 1.470323 \\
Prob(F-statistic) & 0.000000 & & & \\
\hline \hline
\end{tabular}

*Regresión obtenida a través del software EViews-7 